Article

\title{
Discourse Networks and Dual Screening: Analyzing Roles, Content and Motivations in Political Twitter Conversations
}

\author{
Felix Bossner * and Melanie Nagel \\ Department of Politics and Public Administration, University of Konstanz, 78457 Konstanz, Germany; \\ E-Mails: felix.bossner@uni-konstanz.de (F.B.), melanie.nagel@uni-konstanz.de (M.N.) \\ * Corresponding author
}

Submitted: 21 October 2019 | Accepted: 10 February 2020 | Published: 2 June 2020

\begin{abstract}
The increasing relevance of social networking platforms is accompanied by a growing number of studies using digital trace data. However, most studies still lack further understanding of the data-generating process. This analytical gap can be directly attributed to the prevalence of quantitative approaches, as only qualitative work is able to generate these insights. The broad methodological toolset of discourse network analysis addresses this shortcoming as it combines both qualitative and quantitative approaches. The present study therefore employs discourse network analysis in order to (1) determine different user groups' varying role as senders and recipients of targeted online conversations, (2) identify and compare Twitter users' (simultaneous) reference to different forms of conversational Twitter content, and to ( 3 ) asses the motivation of @message authors to direct particular Tweets at particular user groups. To this end, this study analyzes @messages during the $B B C$ program 'Question Time' on 2nd of June 2017-the final media encounter of Prime Minister Theresa May and Jeremy Corbyn in the context of the 2017 UK election campaign. We draw on the theoretical background of Maarten Hajer's discourse coalitions approach in order to investigate the preconditions for the formation of discourse coalitions in new and emerging virtual discourse arenas. Thus, our work not only mirrors the focus in existing literature on Twitter usage during high-profile political media events, but also emphasizes Twitter's unique features for interactive exchange. This article identifies different forms of meta-talk and policy issues, which vary in both their general popularity with Twitter users as well as their interconnectedness. Furthermore, our analysis uncovers the motivation behind the decisions of @message authors to send particular @messages to certain groups of Twitter users. Finally, we could establish that media events only temporarily affect the topical foci of @message authors.
\end{abstract}

\section{Keywords}

discourse network analysis; Jeremy Corbyn; political campaigns; Theresa May; TV debate; Twitter; UK elections

\section{Issue}

This article is part of the issue "Policy Debates and Discourse Network Analysis" edited by Philip Leifeld (University of Essex, UK).

(C) 2020 by the authors; licensee Cogitatio (Lisbon, Portugal). This article is licensed under a Creative Commons Attribution 4.0 International License (CC BY).

\section{Introduction}

The complexity of political processes results to a large degree from the blurriness and subjectivity of perceptions and interpretations which actors adopt during the political and public discussions that precede the decision-making process. The 'argumentative turn' in policy analysis addresses this complexity by explaining policy developments particularly through patterns of collectively constructed perception and argumentation (Janning, Leifeld, Malang, \& Schneider, 2009, p. 59). Drawing on symbolic interactionism, this discursive construction of shared interpretations came to be defined as 'framing' (Entman, 1993; Leifeld \& Haunss, 2012).

Over the last decade, the argumentative turn produced a growing number of literature on policy discourse and discourse networks with several studies focusing on contemporary policy debates to map ideological net- 
works over time (e.g., Fergie, Leifeld, Hawkins, \& Hilton, 2019; Leifeld \& Haunss, 2012). At the outset, most of these studies analyzed contemporary policy issues by primarily relying on data generated from newspaper articles (e.g., Fergie et al., 2019; Leifeld \& Haunss, 2012) or the content of Congressional hearings (e.g., Fisher, Waggle, \& Leifeld, 2013). However, the largely unpredicted results of the US presidential elections and the Brexit referendum in 2016 not only challenged the validity of traditional political science data sources, but also underlined the increasing relevance of social networking platforms for framing political problems and determining the salience of policy issues. This development resulted in a large and constantly increasing body of political science literature, which refers to digital trace data for assessing and predicting collective political opinion formation.

Yet this new source of data also produces newand only partly considered-challenges: In contrast to other fields of study that employ digital trace data, political research on social media is always situated in the tension field between the logics of traditional news reporting and the rationale of 'new media' venues. On the one hand, most of these studies focus on the online deliberation of election campaigns (Ginnis \& Miller, 2017) which attract a particularly large set of contributions during campaign-related 'media events' like TV debates and the announcement of election results (e.g., Ceron \& Splendore, 2019). Thus, the respective studies do not examine 'unique' virtual discourses, but rather investigate practices of 'dual-screening' (e.g., Camaj \& Northup, 2019; Gil de Zúñiga \& Liu, 2017), i.e., how online communities reflect on an agenda set by the traditional media and deliberated by established political elites. On the other hand, the majority of political social media research draws on digital trace data from the microblogging service Twitter. In contrast to its competitors like Facebook or Instagram, Twitter is characterized by its hybrid character between news medium and social network (Chadwick, 2017) as well as by its design as an 'end-user innovation' (Johnson, 2009) that allows its users to develop conversational conventions, which are subsequently embedded in the platform's technological software structure. Thus, Twitter provides its users with multiple modes of content exchange, which either mirror the unidirectional top-down 'broadcast' rationale of traditional news reporting or diverge from this logic and exemplify the 'conversational' interactive and reciprocal nature of social media (cf. Supplementary File): Firstly, Twitter users' decision on whether to add a hashtag to their Tweet implies the choice between just 'broadcasting' their message to their subscribers or sharing their contribution with a-largely unfamiliar-audience that discusses the respective hashtag. Secondly, a Twitter user can react to another user's Tweet by either retweeting-i.e., rebroadcasting-the other user's original messages or by entering a directed conversation with the respective user. For the latter way of interaction, Twitter users integrate another username in the message text-preceded by an @-symboland are thus "able to send directed messages to the mentioned user's inbox" (Borondo, Morales, Benito, \& Losada, 2014, p. 404). While Twitter users mostly stay unaware of being retweeted, they receive a notification when they are mentioned. This increases the probability of direct communication and underlines the conversational aspect of the @message mechanism.

In summary, the confrontation between the topdown broadcasting approach established by traditional political and media elites and social media's conversational bottom-up disruption of that logic should be particularly visible in @message conversations which are delineated by a particular hashtag and concern a particular media event covered by the established media. While some previous works reflect on this tension by comparing the different roles of elite and non-elite Twitter users in 'dual-screening' @message conversations (e.g., Lin, Keegan, Margolin, \& Lazer, 2014), there is a lack of research on why particular user groups engage in political Twitter conversations and what they actually discuss. However, this information is crucial in order to assess if dual-screening Twitter discourse allows the collective formation of frames and discourse coalitions in the first place. Therefore, the present article proposes a new and more comprehensive approach for examining @ messages authors' (1) different roles, (2) simultaneous reference to different topics or storylines, and (3) issuerelated motivation to address particular users during a media event.

\section{Theory, Previous Research and Research Interests}

Hajer (1993) identifies two factors, which support a discourse coalition's success. On the one hand, to feature more frequently in the media allows a discourse coalition to dominate the discourse. On the other hand, attracting a higher number of voters requires the respective coalition to integrate its core frames into a consistent storyline. In particularly important political phases such as election campaigns, these storylines and frames of policy issues are crucial for the formation and sharing of opinions (Kangas, Niemelä, \& Varjonen, 2014) as well as for eventually persuading the voters. Members of a discourse coalition are held together by the construction of a common understanding of the nature of the problem under consideration (Hajer, 1993).

In this context, we aim at providing a starting point to investigate the presence of these processes for the formation and framing of discourse coalitions in 'dualscreening' virtual discourse. More particularly, by zooming in on the directed interaction between Twitter users commenting on a particular media event in context of a particular election campaign, our study examines three crucial preconditions for the actual occurrence of political discourse and the formation of discourse coalitions in Twitter conversations. Firstly, we investigate how the 
configuration of exchange between different groups of users-and thus their capacity to build or influence discourse coalition-in 'new media' diverges from the respective structures in 'old media.' Secondly, we uncover both the actual content of as well as the overlap of content between different Twitter users' discursive contributions. Particularly the latter aspect allows us to assess the most important precondition for the development of shared frames and coalition building, namely if Twitter users actually debate about the same topics and if these topics are integrated into a comprehensive discourse structure. Finally, we investigate if Twitter users distinguish between the recipients of their discursive efforts depending on the respective Tweet's content. We can therefore assess whether Twitter users are generally able to form strategically successful discourse coalitions, which are delimited by particular issues, or instead randomly talk to the 'void.'

\subsection{Roles in @Message Discourse:Who Messages Whom?}

Previous studies that compare the different roles of elite (e.g., politicians, media actors) and non-elite Twitter users (e.g., citizens, bloggers) in 'dual-screening' @message conversations share two main insights (cf. Jungherr, 2015): On the one hand, elite users are able to translate their privileged status as receivers of public attention during media events into the 'Twittersphere' and are therefore more likely to receive @messages than non-elite users. On the other hand, direct interaction with the audience requires 'more commitment' than the simple top-down broadcasting to which political/media elites are more accustomed, which is why they are also less likely to engage in @message conversations than 'average' Twitter users or bloggers. In order to assess different user groups' potential to influence the salience of issues and to form discourse coalitions, our first research question aims at a more fine-grained review of the validity of these findings for the investigated dataset:

RQ1: How does the volume of received and sent $@$ messages during media events differ between elite Twitter user groups (like politicians, parties, journalists and media venues) and non-elite Twitter users (like 'average' citizens and bloggers)?

\subsection{Content of @Message Discourse: What Is the Content of the Messages and How Does It Overlap?}

While as of yet there is no systematic research that focuses on the content of political @messages, some studies have already investigated the issues referenced by Twitter users during TV debates. These works suggest that Tweets can generally be classified as either 'policy debate' or 'meta-talk'-i.e., Tweets, which do not "correspond to any political issue, but [rather constitute] debate about the debate" (Kalsnes, Krumsvik, \&
Storsul, 2014, p. 317). This research also indicates that (particularly non-elite) Twitter users involved in 'dualscreening' prefer meta-talk to policy debates (Freelon \& Karpf, 2015). Our second set of research questions aims at enhancing these insights not only by breaking down both concepts into more fine-grained and inductively derived manifestations and comparing their respective salience in Twitter users' @messages. We also utilize the toolset of DNA to assess if users actually integrate multiple topics into an overarching discourse strategy and evaluate the network structures resulting from the simultaneous reference of Twitter users to these manifestations of content. Thus, we can determine the linkages between and the centrality of different types of content of @messages as well as how likely an @message author who references a particular policy topic or form of metatalk also contributes to another manifestation of policy debate or meta-talk. This enables an assessment of the structural precondition for the development of shared beliefs-and accordingly discourse coalitions. In addition to this, analyzing the policy debate allows us to assess if and how the policy issues discussed in @message conversations mirror the media event's agenda during the course of the debate:

RQ2.1: How do (different manifestations of) policy debate and meta-talk diverge in respect to their general popularity with the senders of @messages? Which policy topics and forms of meta-talk are most often referenced in @messages during TV debates?

RQ2.2: How do different manifestations of policy debate and meta-talk diverge in respect to their linkages to other policy topics and forms of meta-talk? Which policy topics or forms of meta-talk are most and least connected to other categories of @message content? How likely does an author of @messages who references a particular policy topic or form of meta-talk also contribute to another manifestation of policy debate or meta-talk?

RQ2.3: How does the TV debate affect the popularity of policy topics within the @message conversations that accompany the media event?

In contrast to contributions about policy issues, which require at least a minimum level of prior political knowledge or interest, meta-talk does not necessitate particular expertise and instead represents the respective @message authors' immediate individual affective reactions to a media event. Regarding RQ2.2, we therefore (1) expect senders of policy-related @messages to generally focus on one particular topic of interest instead of commenting on multiple policy issues, while (2) Twitter users who engage in a 'meta-talk' Twitter conversations are likely to not only utilize one but multiple forms of 'meta-talk' in their @messages. Furthermore, (3) authors of policy-related statements are accordingly expected to 
be able to participate in meta-talk debates, but not necessarily vice versa. As these users only focus on one or few particular policy topics, it is likely that their affective perception of both the political and the metacharacteristics of the media event depends on the coverage of the issue which interests them the most. Thus, it can be assumed that (4) some forms of meta-talk are more likely associated with a particular set of policy issues than others.

\subsection{Motivation of the Senders of @Messages: Who Receives What Content?}

Investigating the content of @messages (RQ2) can help explain the insights about the diverging roles of different user groups in @message discussions (RQ1): An @message sender's decision to direct their Tweet to a particular recipient is most likely determined by the policy or meta-talk content of their Tweet. Investigating the relationship between the different content foci of Twitter users and the group membership of their preferred addressees (i.e., elite or non-elite user) can therefore contribute to a more detailed understanding of different actor groups' role as @message recipients as well as allow for a better comprehension of @message senders' general discourse motivation. Uncovering this motivation helps to assess if Twitter users select the recipients of their discursive efforts more or less randomly:

RQ3: Is the decision of Twitter users for a recipient of their @message dependent on the content of their contributions?

\section{Case, Data and Methods}

\subsection{Case Selection}

We examine our research questions with regard to @message conversations with the hashtags \#jeremycorbyn and \#corbyn during the $B B C$ program "Question Time" on 2nd of June 2017, which was the final appearance of Prime Minister Theresa May (Conservative Party) and her contender Jeremy Corbyn (Labour Party) in the 2017 UK General Election Campaign. By zooming in on the @message conversation between Twitter users commenting on a particular media event in context of a particular election campaign, our study partly diverges from most of previous political Twitter research: Pinpointing one TV debate allows us to limit our investigation to actual 'dual-screening' behavior, which constitutes the most typical form of political Twitter debate and exemplifies the complex interrelationship between virtual discourse and the roles and content of traditional news reporting. Similarly, focusing on @messages concerning one instead of both candidates narrows the overarching discourse space we examine to a degree, which allows us to investigate if users participating in the same discourse actually talk about the same issues.
We chose to focus on Jeremy Corbyn's election campaign for a number of reasons. Firstly, previous research indicates that Twitter discourse plays a particular crucial role for mobilizing supporters in anti-establishment insurgency campaigns (Jungherr, 2015) like Corbyn's. Secondly, by comprising mainly young, urban and political interested voters, which are highly partisan in their political leanings (e.g., Sayers, 2015), Corbyn's electorate not only reflects the typical characteristics of Twitter users (Jungherr, 2015), but also-by a majority of $57 \%$-utilizes social media as main source of news (Sayers, 2015). Finally, British media's coverage of Jeremy Corbyn is often viewed as particularly divisive (Cammaerts, DeCillia, Magalhães, \& Jiménez-Martínez, 2016), which makes the examination of 'dual-screening' behavior (and thus Twitter's potential role as corrective force) particularly relevant.

The program "Question Time" was the only media event in context of the campaign which resembled a traditional TV debate, as Theresa May refused to take part in a head-to-head debate, but agreed to appear directly after Jeremy Corbyn in the respective TV show. The questions were asked as well by the TV audience in York as by the reporters. The TV broadcast touched upon most of the relevant topics of the election campaign and the storylines put forward by the respective campaigns and the mass media.

\subsection{Data and Coding}

To determine which hashtags are the most valid marker for Twitter debate about Jeremy Corbyn's campaign, the most mentioned hashtags in the UK were analyzed for consecutive three days in advance of data collection. The raw data was retrieved from Twitter's Streaming Application Programming Interface and coded in a twostep procedure: On the one hand, @message senders and recipients were classified into seven distinct groups (media outlets, individual journalists, parties or partyaffiliated organizations, politicians, NGOs, political bloggers and 'average' citizens). On the other hand, the content of the @messages was coded utilizing the discourse network analysis (DNA) approach and software by Leifeld (2017), which allows coding one @message/text portion according to multiple possible categories. Therefore, we can identify multiple different statements in one $@$ message. The respective categories are inductively derived, revised and finally aggregated to higher-level variables that reflect different forms of meta-talk and policy debate.

\subsection{Method}

In order to investigate our first research question, we model a directed one-mode network whose nodes represent senders or recipients of @messages. The nodes are linked to one another if one user's Tweet (sender) mentions another user's account (recipient) 
in their @message. The volumes of received and sent @messages per group are compared by employing descriptive statistics as well as statistical tests to assess the effect of a Twitter user's membership in the coded user groups on the user's indegree and outdegree centrality (i.e., the number of received/sent @messages). For answering our second set of research questions, we utilize descriptive statistics to compare the salience of different manifestations of meta-talk and policy debate as well as the toolset of DNA to identify the overlapping reference to these categories of @message content. To this end, we generate three different one or two-mode concept congruence networks which model the overlapping contributions of @messages authors to the respective forms of content.

Our final research question aims at clarifying the motivation of @message senders by examining if their decision to direct their Tweet to particular recipients is dependent on the policy or meta-talk content of their Tweet. To this end, we created a dataset in which each @message sender constitutes one unit of observation. The first set of variables (i.e., content foci) in this dataset aggregates each users' overall number of statements according to each manifestation of meta-talk or policy debate. Thus, the respective variables capture the number of times that a particular user referred to a particular manifestation of @message content. Similarly, the second set of variables (i.e., choice of recipient) summarizes the number of times each user sent messages to a particular category of recipients. In order to investigate the interrelationship between both sets of variables, we modelled separate regression models for each category of recipients, in which each manifestation of @ message content serves as independent variable and the respective group of recipients represents the respective dependent variable (we run the regression with the same independent variables for each dependent variable). In contrast to a regression model, in which each @ $@$ message serves as unit of observation, the chosen approach ensures the independence of our units of observation and emulates conventional questionnaire-based methods of analysis, which correlate characteristics of users (here: contentrelated foci) and their action (here: choice of recipient).

Thus, we can not only examine if Twitter users choose the recipients of their discursive efforts randomly or on basis of their @message's content, but also evaluate the significance of differences between the content each user group receives. Due to the distribution and characteristics of our data, we applied negative binomial regression.

The network analysis and all visualizations were conducted using the software Visone (Brandes \& Wagner, 2004). All other estimations were conducted in the statistical computing environment $R$ ( $R$ Core Team, 2019) using the packages COUNT (Hilbe, 2016) and MASS (Venables \& Ripley, 2002) for the regression analysis.

\section{Results}

\subsection{Research Interest 1: Roles in @Message Discourse}

To answer our first research question, we disaggregate all $888 @$ messages with the hashtags \#jeremycorbyn or \#corbyn, which were posted during the TV debate according to the coded group identity of their authors and recipients (cf. Supplementary File for full results).

This effort shows that elite actors (i.e., politicians, journalists, media venues and parties) hardly engage in conversational @message exchange, while average citizens (751 Tweets by 497 accounts) and bloggers (93 Tweets by 42 accounts) account for the majority of Twitter conversations. However, this relatively large number of @message senders focuses its conversational efforts on a comparatively small set of other users.

Due to the highly skewed distribution of our data, it is problematic to derive a statistical inference through the average-based method. Therefore, instead of using parametric ANOVA and t-test, we utilized a non-parametric Kruskal-Wallis and Wilcoxon-Box test to assess if there is a statistically significant difference in the number of received@messages among members of different user groups (see Supplementary File). While the results of the Kruskal-Wallis test indicate the general presence of significant differences between the groups $(X 2=49.48$, $p>0.01$ ), the Wilcoxon-Box test assesses the probability that the difference between each pair of groups is a product of randomness. Although the latter analysis indicates that elite users are significantly more likely to receive @messages than citizens $(p<0.01)$, they also imply that the likelihood of politician and party-affiliated accounts to be addresses of @message does not significantly differ from the respective likelihood of bloggers $(p>0.1)$, which in turn are also significantly more likely to receive @messages than citizens $(p<0.01)$. Thereforein regard to our first research question-we can only partially confirm the results of previous research: On the one hand, the investigated @message discourse is almost exclusively ascribable to the conversational activity of bloggers and citizens rather than elite users, while the latter are indeed more likely recipients of these efforts than citizen users. On the other hand, bloggers are not necessarily less likely to receive @messages than some elite groups, which underlines their role as intermediary users between political elites and the 'Twitter population.'

\subsection{Research Interest 2: Content of @Message Discourse}

In summary, the content of the 888 examined @messages amounts to 1.406 statements, of which $504(33.7 \%)$ cover policy issues, while 992 (66.3\%, sent by 485 users) contribute to the meta-talk about the media setting and the appearance of politicians. 


\subsubsection{Meta-Talk}

Regarding the popularity of different meta-talk concepts in @message conversations, Table 1 disaggregates the latter 992 statements according to the different types of meta-talk. This provides more detailed insights into the particular forms of meta-talk and their respective prominence within the conversational Tweets of bloggers and citizens: In line with previous research (Kalsnes et al., 2014), this analysis identifies Twitter users' personal evaluation of the TV appearance of politicians as the most dominant form of meta-talk (432 statements by 226 users). However -in contrast to the expectations of previous studies - Twitter users more visibly evaluate the professional competency of the contestants (251 statements by 178 users) than they discuss the tone and style of their appearance (181 statements by 147 users).

The second most referred overarching category of meta-talk ('general comments') subsumes multiple different types of commentary which share a more or less distinct detachment from the TV debate's immediate context. The most dominant manifestation of this type of meta-talk (partisan statements) underlines the high degree of polarization in Twitter discourse (Jungherr, 2015): The relative balance between satirical or personal anecdotes and fact checking indicates that Twitter conversations equally constitute an arena for serious policy debates and a 'virtual fireside' for more trivial chats. The comparatively low amount of general 'anti-establishment' statements could partly result from Jeremy Corbyn's image as 'anti-establishment candidate,' because of which users refrain from general criticism and instead take sides with the non-mainstream contestant. The third most popular type of meta-talk is idiosyncratic for dual-screening as it embeds the virtual evaluation of the TV debate in other simultaneously occurring 'old' or 'new' media discourses by linking the Twitter debate either to the TV debate itself or to other second-screen media venues. By criticizing the latter's content, the largest number of venue-linking statements expands the meta-debate about the immediate TV show to an even larger debate about the 'new' and 'old' media setting of the election campaign in general ('metameta-debate'). This observation is particularly noteworthy because the evaluation of the TV debate's immediate setting is the least prominent form of meta-talk. Unsurprisingly, only a marginal number of users praises the moderators, audience or the format of the show (14 statements by 12 users), while the majority of conversational contributions criticizes the media event's setting (132 statements by 86 users). This underlines the predominantly negative tone of Twitter content in general (Jungherr, 2015).

Regarding network structure of meta-talk in @message conversations, our research approach not only aims at uncovering the diverging salience of different (inductively derived) types of meta-talk (RQ 2.1), but also at assessing the structural precondition for the development of shared beliefs, integrated discourse strategy and discourse coalitions, i.e., if and how users simultaneously refer to multiple manifestations of meta-talk (RQ 2.2). While the user counts in Table 1 indicate that the majority of users within the meta-talk discourse indeed refer to more than one particular form of meta-talk, we switch to a network perspective in order to draw a more comprehensive picture of the integration of different meta-talk manifestations. To this end, Figure 1 visualizes the concept-congruence network of the respective Twitter debate. This network's nodes represent the different types of meta-talk and are linked if a user simultaneously utilizes two concepts (with the size of the edges indicating the respective number of users).

In general, the presented network indicates that meta-talk contributions of @message authors result in an integrated, comprehensive and densely connected debate structure, as all nodes are at least indirectly connected to each other and each node is connected to at least two nodes of a different type of meta-talk (cf. node

Table 1. Number of statements and users for each meta-talk concept.

\begin{tabular}{ll}
\hline Topic (statements/users) & Meta-talk concept (statements/users) \\
\hline Evaluation of TV debate media setting (146/96) & Praise for media setting (14/12) \\
& Criticism of media setting (132/86) \\
Linking TV debate to other venues (158/118) & Link/General reference to TV debate (26/22) \\
& Link/General reference to other second screen medium (54/39) \\
& Criticism of other second screen mediums (78/64)
\end{tabular}

Evaluation of politicians (432/226)

Evaluation of politicians' style and tone (181/147)

Evaluation of politicians' competency (251/178)

General comments (259/179)

Fact checking (69/52)

General partisan statements (103/83)

Satire and personal anecdotes (55/45)

General anti-establishment statements (32/24)

Notes: The first count indicates the number of statements identified for each type or manifestation of meta-talk, while the second count indicates the number of users contributing to the respective concept or topic. 


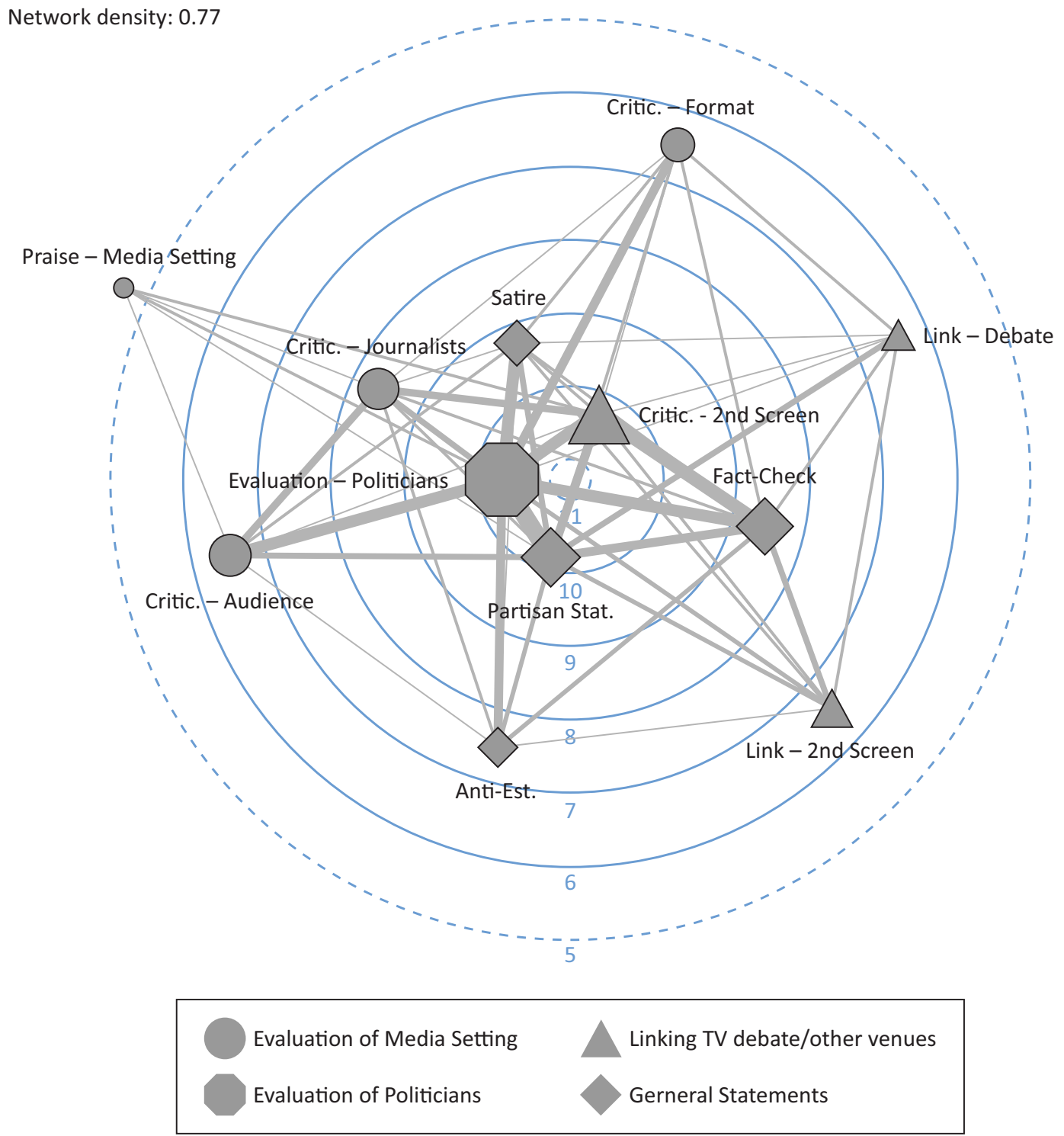

Figure 1. One-mode discourse network of meta-talk conversations. Notes: The edge width visualizes the number of @message senders which refer to both categories of content. The position of a node in the radial layout indicates number of other manifestations of content referred to by users contributing to the respective concept. The node size visualizes the overall number of users referring to at least one other than the respective concepts. The node shapes are as follows: Circles denote meta-talk evaluating the media setting of the debate; octagons denote meta-talk evaluating the debate performance of politicians; triangles denote 'venue-linking' meta-talk; diamonds denote general meta-talk comments; squares denote manifestations of policy debate.

type, caption of Figure 1). Accordingly, Twitter users who use one type of meta-talk are also likely to engage in other forms of meta-talk.

The network's three core nodes-'partisan statements,' 'criticism of second-screen media' and 'evaluation of politicians appearance' - are linked to all 11 other concepts of the network and reflect three of the four overarching types of meta-talk (cf. node types). Thus, they additionally accentuate the network's embeddedness: By taking side with one politician, evaluating her or his opponent's performance and/or scrutinizing the content of second-screen media, @message senders generate an overarching frame of reference which allows to posit, compare and link their other meta-talk contributions. The strong linkage of these nodes themselves suggests that Twitter users either base their assessment of second-screen media on the perception of the politicians' performance or vice versa, while both are either derived from or significantly influence their respective partisan support.

The equally weak linkage of references to the TV debate and references to second-screen media partly uncovers the dynamics of @message conversations: There is obviously only a small set of users who initially reference other second-screen venues in context of a limited amount of meta-talk issues. However-as the central 
position of second-screen criticism underlines-a larger number of users picks up these links during the course of the debate and transcends their scope by scrutinizing their content concerning a broader set of concepts.

Regarding general statements, both 'satire' and 'factchecking' are in a clear proximity to the network core. The former's linkage to nearly all (except one) other concepts underlines the crucial role of satire for all types of (meta)discourse on Twitter, while the latter's strong connection to the three cores nodes indicates its relevance as an instrument for increasing the persuasiveness of Twitter users' general convictions or their judgements about politicians and second-screen media reports.

\subsubsection{Policy Debate}

Regarding the popularity of different policy issues in @message conversations, Table 2 disaggregates the 504 policy-related statements and their 245 authors according to 10 inductively coded concepts. This overview indicates that security (predominantly Corbyn's position on nuclear weapons) is the by far most prominent topic in the @message conversations, followed by the discussion of historical political events (mostly the Irish peace process), economic policies (mostly austerity policy and the cost of Labour's election pledges) and questions of political strategy (mainly May's decision for a 'snap election'). Although all topics of the TV debate are represented in the Twitter discourse, the varying degree of attention to specific issues illustrates that Twitter discourse does not necessarily just reflect the agenda setting of traditional media venues. On the contrary, 'traditional' domestic sociopolitical policy issues like the changes in society and social discourse, the labor market, health and education and migration received much less attention in the @messages of Twitter users than in the TV debate. This finding is particularly remarkable as it contradicts the assumption of previous literature that Twitter users are more likely to comment on topics or events which affect them personally than on abstract geopolitical or power-related issues. With respect to the specific circumstances of the 2017 UK General Election, it is at least surprising that the discussants' position on Brexit and the EU played only a minor role in the @message debate.
Regarding the effect of media event on policy debate in @message conversations, in order to assess if the respective issues' salience is a direct reaction to the established media's coverage or if it follows a partially diverging logic (RQ 2.3), Figure 2 visualizes the distribution of @message statements per topic over time (in 10 minute frequencies) during, shortly before and after the TV show (20:20-21:30), and produces three key insights: Firstly, the four most prominent issues (history, security, economy, and political strategy) already represented the largest share of the online debate before the TV program started. In all the cases, the TV debate's start leads to a temporary decline in topic-specific attention, which is overcome as soon as the topic is picked up in the program and fades after the end of the TV show. Secondly, the overall less prominent 'traditional' sociopolitical policy topics (labor market, migration, health and education, society) were scarcely debated before the TV debate's start, which temporarily called attention to these issues, but vanished after the end of the debate. Thirdly, Brexit was one of the most discussed issues before the TV debate, which surprisingly shifted attention away from the topic, change still observable after the debate. Thus, in respect to most topics, media events like TV debates only have a limited and temporary influence on the logic of agenda setting on Twitter, while in some (exceptional) cases like the Brexit debate the 'elite' media environment is indeed able to generate sustainable shifts of the @message agenda.

Regarding network structure of policy debate in @message conversations, similar to Figure 1, Figure 3 depicts the discourse network emerging from the simultaneous reference of @message senders to multiple policy issues in order to assess the integration of policy debate and the potential for coalition building (RQ 2.2). In contrast to the meta-talk network, the policy debate does not produce an integrated network, as the node 'environment' is isolated from the rest of the nodes. This indicates that the four users discussing environmental policies do not refer to any other policy issues. However, the other nodes of the discourse network are not only as integrated as the meta-talk concepts, but also more densely connected with each other. When ignoring the unconnected debate about environmental policies, $89 \%$ of all

Table 2. Number of statements per policy issue.

\begin{tabular}{lc}
\hline Policy issue & Number of statements (users) \\
\hline Security & $174(115)$ \\
Economy & $77(45)$ \\
Historical political events & $77(34)$ \\
Political strategy & $58(39)$ \\
EU-membership of the UK/Brexit & $41(29)$ \\
Society & $31(20)$ \\
Labor market & $17(12)$ \\
Health/Education & $14(12)$ \\
Immigration & $10(6)$ \\
Environmental policy & $5(4)$ \\
\hline
\end{tabular}




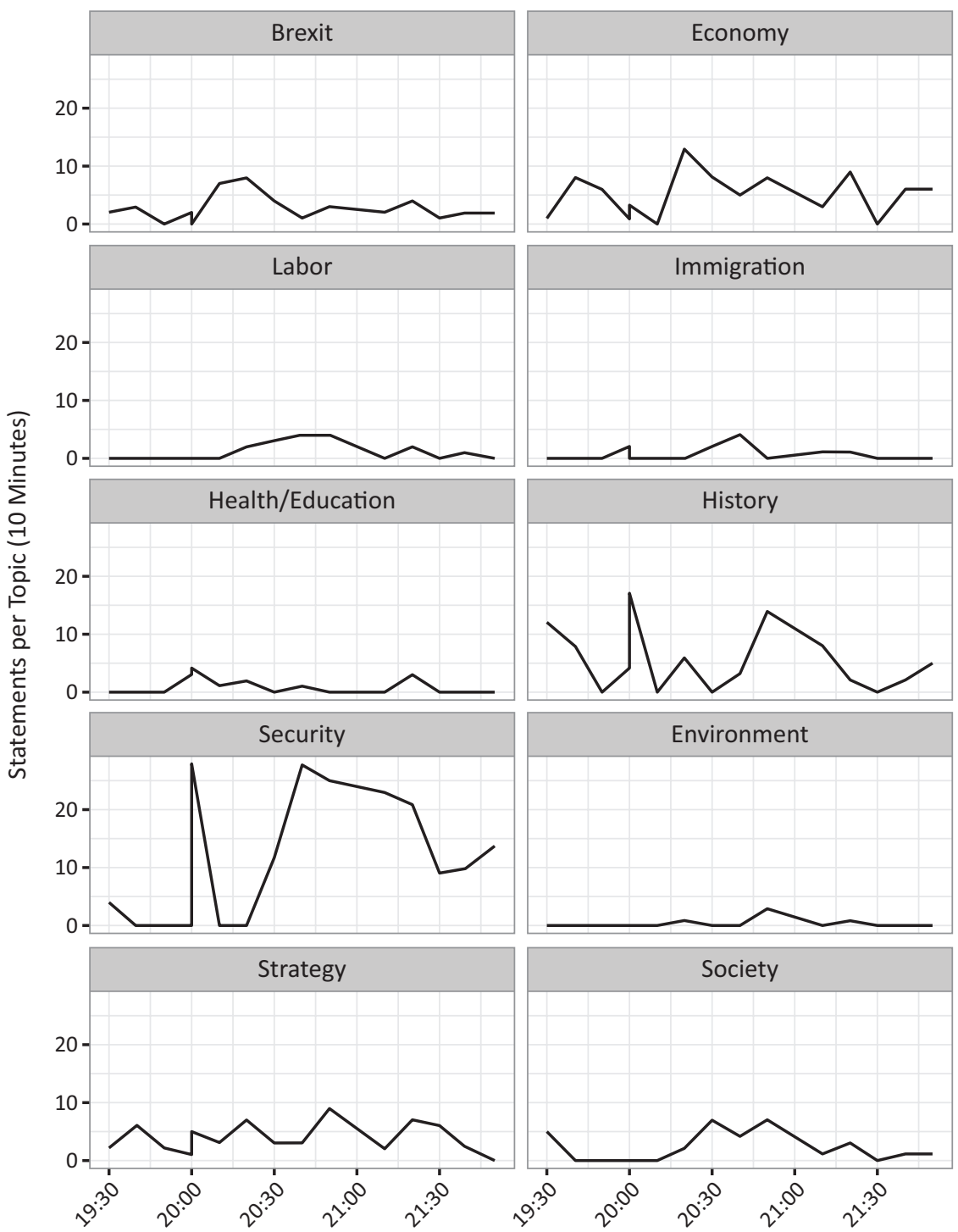

Figure 2. Volume of policy issue statements over time during TV debate. Notes: Each time series graph visualizes the course of the number of statements associated with each policy topic in 10-minute intervals during, shortly before and after the TV show (20:20-21:30).

potential connections within the network are covered by the actual observable linkages between the policy issues. This implies that @message authors relate policy issues more immediately with each other than they link different forms of meta-talk.

In contrast to the meta-talk network, the prominent position of the policy debates' core nodes does not necessarily reflect their overall popularity with @message authors (cf. Table 2): Although security issues are more often discussed (115) than economic policies (45), both concepts are linked to the same number of other statements. Furthermore, the unanticipated secondary role of Brexit and society in respect to the overall distribution of users' attention is contrasted by their central position in the discourse network. Thus, although being an overall less popular matter of debate, both topics constitute important reference points through which some users locate and interpret all other political concepts.
If we consider the strength of the lines between the most dominant issues (security, economy, history, strategy and Brexit), the previously discussed bifurcation of the discourse is visible. The dominant issues are more strongly connected to each other than to sociopolitical issues, while the latter are more evenly linked to (nearly) all of the remaining policy discussions. Thus, the domination of (historical) foreign policy-related, economical or strategical issues is also represented in the relational expression of the conversational efforts of @message authors.

\subsubsection{Links between Meta-Talk and Policy Debates}

For a comprehensive assessment of discourse integration and coalition potential, the previous isolated relational analyses of meta-talk (cf. Figure 1) and policy debate (cf. Figure 3) must be complemented by an examina- 
Network density: 0.71

Network density (without Envir.): 0.89

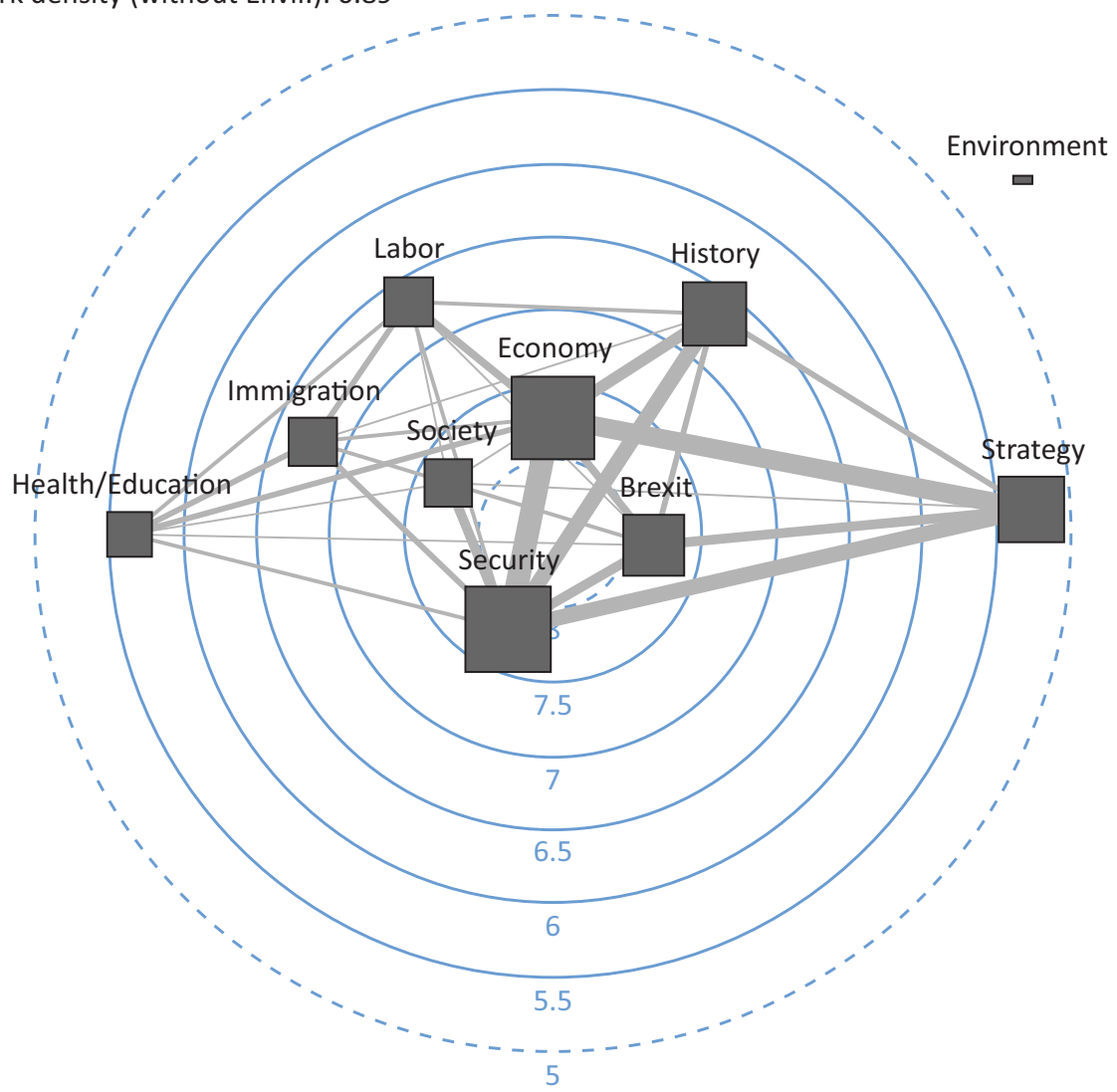

Figure 3. One-mode discourse network of policy debate. The edge width visualizes the number of @message senders which refer to both categories of content. The position of a node in the radial layout indicates number of other manifestations of content referred to by users contributing to the respective concept. The node size visualizes the overall number of users referring to at least one other than the respective concepts. Squares denote manifestations of policy debate.

tion of links between the manifestations of both types of @ message content. To this end, Figure 4 visualizes a twomode discourse network, in which an edge between two nodes is only modelled if a user simultaneously refers to a policy (dark grey nodes) and a meta-talk concept (light grey nodes).

The network's overall integrated structure indicates that each policy issue is associated with a number of particular meta-talk issues and vice versa. Thus, it can be concluded that both forms of content are embedded in an overarching integrated debate context. As the size of the policy-related nodes equal the respective node size in Figure 3 , all of the users discussing a policy issue also refer to at least one meta-talk concept. The same is not true for the authors of meta-talk @messages, as the size of the meta-talk nodes in the two-mode network is smaller than in Figure 1. This validates that contributing meta-talk @messages require less political expertise and interest than the participation in policy debates, which is why authors of policy-related messages are also likely to discuss meta-talk concepts but not vice versa. In this context, the discursive embeddedness of environmental policies is particularly noteworthy: Albeit users discussing this topic do not refer to any other pol- icy issue (cf. Figure 2), they participate in the debate on some meta-talk concepts. Accordingly, meta-talk serves as a point of connection for indirectly linking the otherwise isolated sub-discussion to the larger context and the deliberation of other policy issues. As all nodes differ concerning their position, size and the strength of their linkage to other concepts, it can be concluded that some policy issues are indeed more likely associated with particular forms of meta-talk (for a more detailed analysis, cf. Supplementary File).

\subsection{Research Interest 3: Motivation of @Message Senders}

Our final research question aims at examining if the decision of Twitter users for a recipient of his @message is dependent on the content of their contributions. To this end, we run six separate negative binomial regression models for each category of recipients, in which each manifestation of @message content serves as independent variable and the respective group of recipients represents the respective dependent variable (see Section 3 for details and Supplementary File for the results). For a comprehensive interpretation, we summarized the re- 


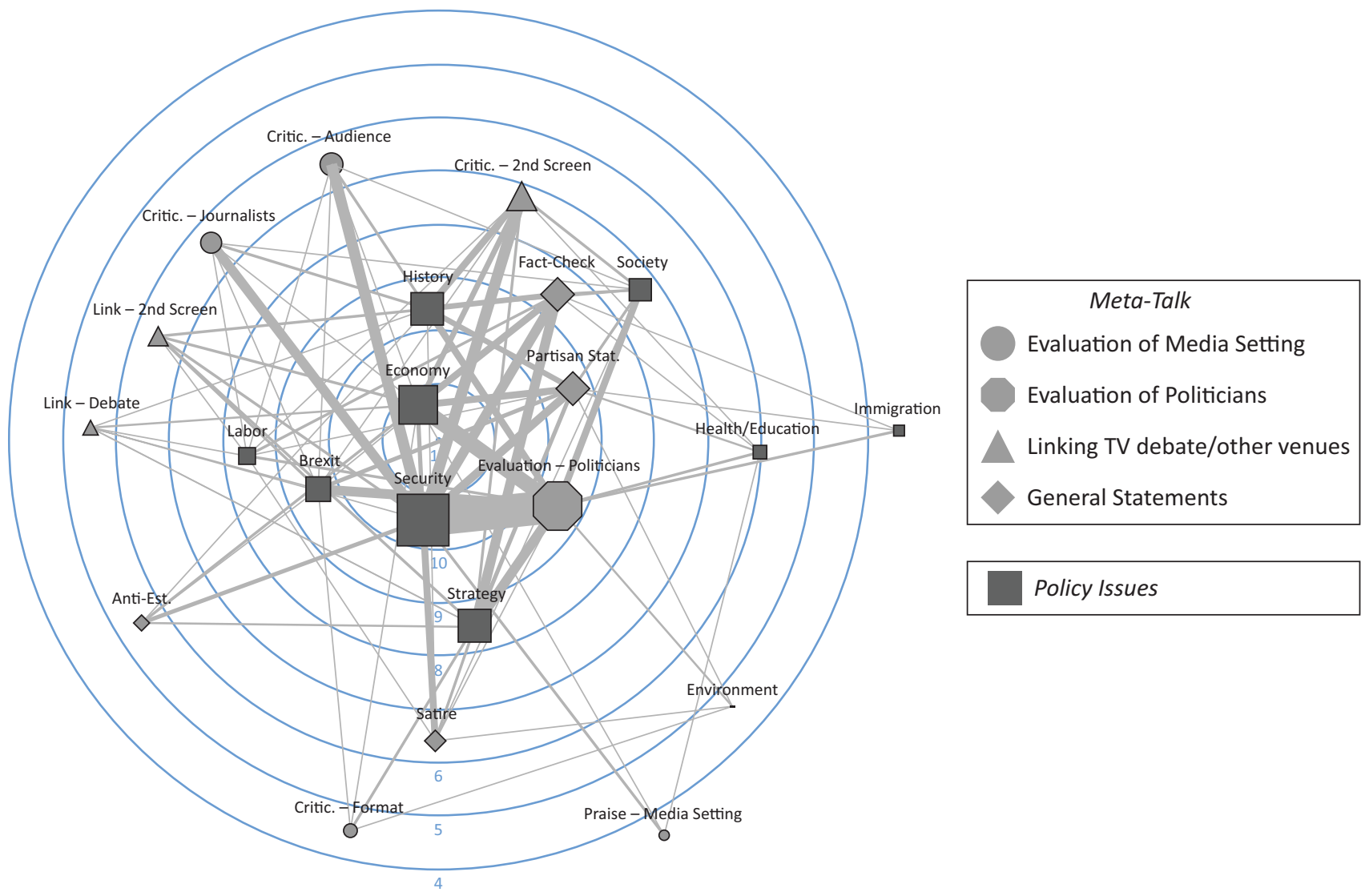

Figure 4. Two-mode discourse network of policy-debate and meta-talk in @message conversations. Notes: The edge width visualizes the number of @message senders which refer to both categories of content. The position of a node in the radial layout indicates the number of other manifestations of the other category of content mentioned by the respective authors. The node size visualizes the overall number of users referring to at least one concept of the respectively other category. The node shapes are as follows: Circles denote meta-talk evaluating the media setting of the debate; octagons denote meta-talk evaluating the debate performance of politicians; triangles denote 'venue-linking' meta-talk; diamonds denote general meta-talk comments; squares denote policy issues.

sults of all regression models in Figure 5. The light grey (meta-talk issues) and dark grey (policy issues) nodes represent independent variables with significant effects in at least one of the regression models-i.e., manifestations of @message content which increase a user's likelihood to choose a particular recipient for their @message. Black circular nodes depict @message recipients (i.e., dependent variables), whose likelihood to be addressed is significantly associated with users' content foci.

In general, this visualization confirms the effect of @message content on the decision of @message senders for the respective Tweet's recipient: The mere presence of edges indicates that some categories of recipients are indeed more likely to be addressed by another user, if this user has more interest in particular policy issues or distinct forms of meta-talk. Therefore, it can be concluded that Twitter users' decision for a recipient of their @message is indeed partially dependent on their contribution's content. The presented network's structure underlines this finding, as the majority of topics is only connected to one category of @message recipients and only one topic ('criticism of journalists') links more than two user groups. However, some concepts (society, links to the debate, health and education) are not at all associated with users' decision to address particular user groups.

More particularly, policy issues (black squares) seem to play only a negligible role in explaining Twitter users' decision to address elite users. Remarkably, the likelihood of @message senders to address politicians and journalists is not dependent on any emphasis on a specific political issue, while parties are only associated with one form of political content and the linkage of media venues to three policy topics is only weakly significant. In contrast to this, non-elite actors like bloggers and citizens are very likely to be contacted by users with clearly delineated political foci. Furthermore (with the exception of political strategy), the preferred policy topics of users who contact citizens and the policy emphasis of users who address bloggers are non-overlapping. This means that Twitter users consciously distinguish between non-elite 'citizen' users and semi-elite bloggers (cf. Section 4.1) as suitable recipients for the respective topics: A user who contacts 'citizens' is more likely to 

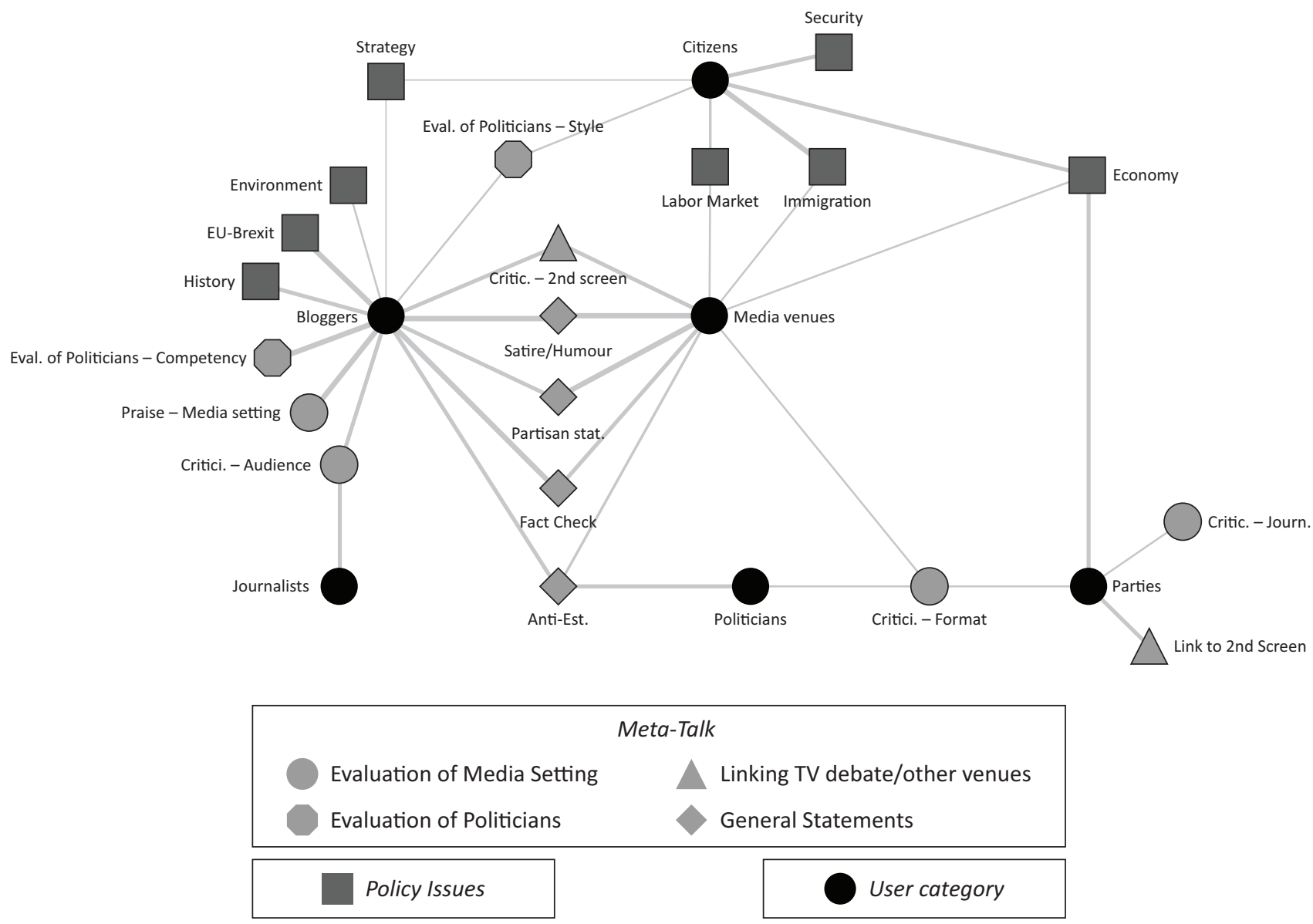

Figure 5. Significant results of negative binominal regression analysis. Notes: The dependent variable is the user category of @message recipients (black nodes); the independent variable is the type of content (grey nodes, node shapes and shades as in Figure 1). The edge width visualizes the level of significance $(p<0.001, p<0.01, p<0$. 05). Only significant coefficients were mapped as nodes.

contribute to 'standard' political topics like the economy, immigration, political strategy and security, while a user who confronts political bloggers is more likely to prefer conversations about context and election-specific issues like the Brexit debate and Jeremy Corbyn's role in the Irish peace process.

The role of @messages that evaluate the appearance of politicians in the debate mirrors this relationship: On the one hand, none of the elite recipients are associated with a particularly high likelihood to receive such @messages, while both bloggers and citizens are likely to receive Tweets by users that emphasize the tone and style of the politicians' appearance. On the other hand, users who confront bloggers also emphasize the competency of the debates' contestants, while users who address citizens do not show a particular preference for that topic. This confirms our previous observation that @message senders consciously distinguish between citizens and bloggers. In a similar vein, citizens are not associated with any form of meta-talk that evaluates the media setting.

Regarding the role of criticism of second screen media as well as fact-checking, satirical, partisan or anti-establishment statements (i.e., 'general statements,' light-grey octagons), bloggers and media venues are structurally equivalent, as both groups are very likely to receive @messages by users who put an emphasis on this form of debate contribution. In contrast to this, politicians, parties and journalists are linked to a maximum of three particular manifestations of content, as most of the respective regressions' explanatory variables were insignificant in our models. This means users who address these elite recipients seem to have less clearly delineated areas of focus, as most forms of content are associated with the same (and accordingly insignificant) likelihood to be directed at the respective user group. Despite profiting from a comparable or even larger amount of conversational attention as bloggers (cf. Section 4.1), elite users therefore receive less targeted @messages as well as@messages that are less distinguishable in terms of their content. This difference could result from the realization of @message senders that elite actors use the @message functionality only reluctantly (cf. Section 4.1). Therefore, users who have a more targeted area of interest and aim at starting a conversation direct their messages at bloggers who combine the more proactive 
Twitter behavior typical for 'citizens' and the more central position in the Twitter attention network typical for elite users (cf. Section 4.1). As receiving more focused @messages also enhances the ability of bloggers to respond to the respective Tweets, these insights underline the crucial role of bloggers as challengers of the agendasetting powers of political and media elites.

\section{Discussion and Conclusion}

From a theoretical perspective, the main aim of our study is to provide a starting-point for investigating the presence of discursive coalition-forming and framing processes in 'dual-screening' virtual discourse. As political research in the field of social media has not yet developed its own theory (e.g., Jungherr, Schoen, Posegga, \& Jürgens, 2017), we base our effort on the discoursecoalition concept put forward by Maarten Hajer (1993) and examine the preconditions for the actual formation of shared beliefs and discourse coalitions in Twitter conversations. While most previous studies utilizing digital trace data quantitatively interpret the salience of different hashtags, keywords or users as indicators for the formation and sharing of opinions, we take a step back and examine if the actual content and patterns of Twitter users' interactions allows for this kind of interpretation. Thus, we can judge whether Twitter conversation generally allows users to form strategically successful discourse coalitions that are delimited by certain topics.

To this end, we first compare the different roles of political or media elites and non-elite Twitter users in @message conversations: In line with previous findings, we confirm elite users' lower likelihood to send @messages in comparison with citizens and bloggers and their higher likelihood to be addressees of conversational attention in comparison with citizens. However, we do not find differences in elite users' and political bloggers' likelihood to receive @messages. This underlines the role of bloggers as intermediaries between conversational unengaged, but heavily contacted political elites and the general 'Twitter population' that actively participates in conversations but is only seldomly contacted. In other words, bloggers are crucial actors for forming and maintaining discourse coalitions relying on Twitter exchange and should therefore receive particular consideration in future more comprehensive research efforts.

Secondly, as one of the as of yet first systematic and comprehensive analysis of @message content during media events, this study embeds previous studies' (e.g., Kalsnes et al., 2014) rather exploratory investigations of the content of Tweets into a more differentiated perspective that uncovers the relational structures resulting from Twitter users' simultaneous reference to multiple forms of meta-talk or multiple policy issues. Utilizing the toolset of DNA, we could substantiate not only that Twitter users involved in a particular discourse actually talk about the same topics and integrate multiple issues into an overarching 'meta-talk' and policy discourse, but also illustrate that some @message content is more likely related to other forms of meta-talk or policy issues than other manifestations of content. As this diverging degree of connectedness did not necessarily mirror the respective concepts' salience, future studies should address this issue in a more detailed manner-particularly with regard to the role of core frames and consistent storylines for the success of discourse coalitions (cf. Section 1).

Finally, we provide a first explanation for the varying distribution of @message attention between elite and non-elite users as we uncover that the decision of @message authors for a recipient of their @message is not random, but dependent on the content of their Tweet. This demonstrates that Twitter users do not talk to the 'void,' but are generally able to consciously or unconsciously address particular users in regard to particular questions and could therefore indeed form discourse coalitions. Regarding the applicability of Hajer's discourse coalitions theory to Twitter conversations, these findings point to two main implications. On the one hand, our results show that @message discourse fulfills crucial preconditions for the actual occurrence of political discourse and the formation of discourse coalitions in Twitter conversations: Twitter users should not only be able to develop shared beliefs and storylines, as their @messages simultaneously refer to and integrate multiple manifestations of content, but could also form discourse coalitions, as they choose the addressees of their Tweets strategically. On the other hand, the crucial position of bloggers (cf. Sections 4.1 and 4.3 ) as well as the difference between the agenda of the media event and the Twitter conversation accompanying it (cf. Section 4.2.2) shows that Twitter discourse follows a partially different logic than traditional discourse arenas: The interaction and mutual influence of political and (broadcasting top-down) media elites (Hajer, 2009) is increasingly challenged by competing agenda-setters like bloggers, which therefore deserve more academic attention.

From a methodological point of view, the present article's main and as of yet unique contribution to previous political research on social media consists in employing DNA and thereby supplementing this work's quantitative insights by a qualitative standpoint. This change of perspective not only embodies a significant divergence from previous - predominantly quantitatively orientedpolitical Twitter research, but also constitutes a promising opportunity to directly address the former's central analytical gaps, as the broad methodological toolset provided by DNA combines the advantages of both qualitative and quantitative methods. Thus, this article should only represent the starting point for applying DNA to political Twitter research-particularly because the exploratory nature of this article entails two severe limitations: On the one hand, our approach differs from most applications of DNA by not identifying and comparing actual shared beliefs and the resulting different discourse coalitions or their respective interrelationships. Instead, we employed DNA to substantiate the precondi- 
tions for discourse coalition formation in Twitter conversation. Considering the success of this approach, we recommend future research to employ a more traditional DNA-approach and investigate the actual presence of shared perceptions and discourse coalitions in virtual debate. On the other hand, we draw on previous studies in the field of political Twitter research by consciously zooming in on the @message conversation between Twitter users commenting on a particular media event in context of a particular election campaign. Future studies should examine the validity of our insights in more comparative research settings.

\section{Acknowledgments}

We want to thank Keiichi Satoh, the Academic Editor and three anonymous reviewers whose constructive comments have greatly improved the article.

\section{Conflict of Interests}

The authors declare no conflict of interests.

\section{Supplementary Material}

Supplementary material for this article is available online in the format provided by the authors (unedited).

\section{References}

Borondo, J., Morales, A. J., Benito, R. M., \& Losada, J. C. (2014). Mapping the online communication patterns of political conversations. Physica A: Statistical Mechanics and its Applications, 414, 403-413.

Brandes, U., \& Wagner, D. (2004). Analysis and visualization of social networks. In M. Jünger \& P. Mutzel (Eds.), Graph drawing software (pp. 321-340). Berlin and Heidelberg: Springer.

Camaj, L., \& Northup, T. (2019). Dual-screening the candidate image during presidential debates: The moderating role of Twitter and need to evaluate for the effects on candidate perceptions. Journal of Broadcasting \& Electronic Media, 63(1), 20-38.

Cammaerts, B., DeCillia, B., Magalhães, J., \& JiménezMartínez, C. (2016). Journalistic representations of Jeremy Corbyn in the British Press: From watchdog to attackdog. London School of Economics and Political Science. Retrieved from http://www.Ise.ac.uk/ media-and-communications/research/researchprojects/representations-of-jeremy-corbyn

Ceron, A., \& Splendore, S. (2019). 'Cheap talk'? Second screening and the irrelevance of TV political debates. Journalism, 20(8), 1108-1123.

Chadwick, A. (2017). The hybrid media system: Politics and power. New York, NY: Oxford University Press.

Entman, R. M. (1993). Framing: Toward clarification of a fractured paradigm. Journal of communication, 43(4), 51-58.
Fergie, G., Leifeld, P., Hawkins, B., \& Hilton, S. (2019). Mapping discourse coalitions in the minimum unit pricing for alcohol debate: A discourse network analysis of UK newspaper coverage. Addiction, 114(4), 741-753.

Fisher, D. R., Waggle, J., \& Leifeld, P. (2013). Where does political polarization come from? Locating polarization within the US climate change debate. American Behavioral Scientist, 57(1), 70-92.

Freelon, D., \& Karpf, D. (2015). Of big birds and bayonets: Hybrid Twitter interactivity in the 2012 presidential debates. Information, Communication \& Society, 18(4), 390-406.

Gil de Zúñiga, H., \& Liu, J. H. (2017). Second screening politics in the social media sphere: Advancing research on dual screen use in political communication with evidence from 20 countries. Journal of Broadcasting \& Electronic Media, 61(2), 193-219.

Ginnis, S., \& Miller, C. (2017). \# GE2015: The General Election on Twitter. In D. Wring, R. Mortimore, \& S. Atkinson (Eds.), Political Communication in Britain (pp. 315-328). Cham: Palgrave Macmillan.

Hajer, M. A. (1993). Discourse coalitions and the institutionalization of practice: The case of acid rain in Britain. In F. Fischer \& J. Forester (Eds.), The argumentative turn in policy analysis and planning (pp. 43-76). Durham and London: Duke University Press.

Hajer, M. A. (2009). Authoritative governance: Policy making in the age of mediatization. New York, NY: Oxford University Press.

Hilbe, J. M. (2016). COUNT: Functions, data and code for count data. CRAN.R Project. Retrieved from https:// CRAN.R-project.org/package $=$ count

Janning, F., Leifeld, P., Malang, T., \& Schneider, V. (2009). Diskursnetzwerkanalyse: Überlegungen zur Theoriebildung und Methodik [Discourse Network Analysis: Reflections on theory formation and methodology] In V. Schneider, F. Janning, P. Leifeld, \& T. Malang (Eds.), Politiknetzwerke: Modelle, Anwendungen und Visualisierungen [Policy Networks: Models, applications and visualisations] (pp. 59-92). Wiesbaden: VS Verlag für Sozialwissenschaften.

Johnson, B. (2009, July 15). Inside Twitter HQ. The Guardian. Retrieved from https://www.theguardian. com/technology/2009/jul/15/twitter-headquartersbiz-stone

Jungherr, A. (2015). Twitter use in election campaigns: A systematic literature review. Journal of Information Technology \& Politics, 13(1), 72-91.

Jungherr, A., Schoen, H., Posegga, O., \& Jürgens, P. (2017). Digital trace data in the study of public opinion. Social Science Computer Review, 35(3), 336-356.

Kalsnes, B., Krumsvik, A. H., \& Storsul, T. (2014). Social media as a political backchannel. Aslib Journal of Information Management, 66(3), 313-328.

Kangas, O. E., Niemelä, M., \& Varjonen, S. (2014). When and why do ideas matter? The influence of framing 
on opinion formation and policy change. European Political Science Review, 6(1), 73-92.

Leifeld, P. (2017). Discourse network analysis. In M. Lubell \& J. N. Victor (Eds.), The Oxford handbook of political networks (pp. 301-326). New York, NY: Oxford University Press.

Leifeld, P., \& Haunss, S. (2012). Political discourse networks and the conflict over software patents in Europe. European Journal of Political Research, 51(3), 382-409.

Lin, Y. R., Keegan, B., Margolin, D., \& Lazer, D. (2014). Rising tides or rising stars? Dynamics of shared attention on Twitter during media events. PloS One, 9(5). https://doi.org/10.1371/journal.pone.0094093

R Core Team (2019). R: A language and environment for statistical computing. $R$ Foundation for Statistical Computing. Retrieved from https://www.r-project. org

Sayers, F. (2015). "You may say that I'm a dreamer": Inside the mindset of Jeremy Corbyn's supporters. YouGov. Retrieved from https://yougov.co.uk/ topics/politics/articles-reports/2015/08/27/youmay-say-im-dreamer-inside-mindset-jeremy-corby

Venables, W. N., \& Ripley, B. D. (2002). Modern applied statistics with S (4th ed.). New York, NY: Springer.

\section{About the Authors}

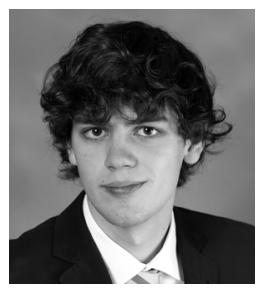

Felix Bossner is a Master Student at the University of Konstanz. His Master thesis is about (discourse) networks in anti-establishment Twitter conversations. His research interests include social network analysis, discourse analysis, social media discourses, digital governance and project management.

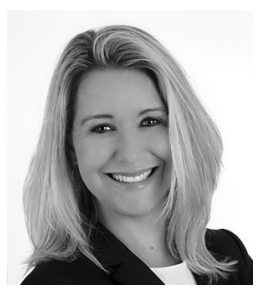

Melanie Nagel is a Postdoctoral Researcher at the University of Konstanz and has recently joined the Institute of Political Science at the University of Tübingen. Her research interests include discourse analysis, social network analysis, natural resource management, climate change policy, infrastructure policy and urban studies. She wrote her dissertation about the discourse over the infrastructure project Stuttgart 21 and published in peer-reviewed journals such as Urban Studies and Urban Climate. 